\title{
Focused Ion Beam Nanotomography of Chondritic Meteorites: Closing the Mesoscale Length Gap in Paleomagnetic Studies
}

\author{
Joshua F. Einsle ${ }^{1}$, Roger R. Fu ${ }^{2}$, Benjamin P. Weiss ${ }^{2}$, Takeshi Kasama ${ }^{3}$, Karl Fabian ${ }^{4}$, Pádraig Ó \\ Conbhuí ${ }^{5}$, Wyn Williams ${ }^{5}$, Paul Midgley ${ }^{6}$, Richard J. Harrison ${ }^{1}$ \\ 1. Department of Earth Sciences, University of Cambridge, Cambridge UK, \\ 2. Department of Earth, Atmospheric and Planetary Sciences, MIT, Cambridge, MA, USA, \\ 3. Center for Electron Nanoscopy, Technical University of Denmark, Kongens Lyngby, Denmark, \\ 4. Geological Survey of Norway, Trondheim, Norway, \\ 5. School of GeoSciences, University of Edinburgh, Edinburgh, UK, \\ ${ }^{6}$ Department of Materials Science and Metallurgy, University of Cambridge, Cambridge UK.
}

One of the greatest limits on the reliability of paleomagnetic information comes from the decreasing fidelity of signal with increasing age of the rock. The older the rock, the more complex is its geological history, and the more likely it is to have experienced conditions that altered, or even destroyed, the primary magnetic signal. This problem gets dramatically worse when it comes to extraterrestrial materials, such as meteorites, that predate the formation of the Earth itself. Nanoscale magnetic minerals sitting inside single silicate crystal hosts carry the most reliable paleomagnetic signals in meteorites. These small magnetic carriers adopt highly stable magnetisation states and are protected from alteration by their surrounding silicate. To interpret such paleomagnetic measurements, and assess their reliability, we need to relate the macroscopic remanence observations to the underlying magnetic properties of individual magnetic particles and to the collective magnetic behavior of the particle ensemble as a whole. Here we outline a combined experimental and computational methodology to quantify the 3D properties of magnetic particle ensembles at the mesoscopic length scale. Electron holography and tomography of individual particles are combined with FIB slice-and-view tomography to provide a complete characterization of the particle ensemble in terms of the domain state, volume, shape and interparticle spacing. This information is used as input to finite-element micromagnetic and kinetic Monte Carlo simulations to obtain a quantitative understanding of the system's response to changes in magnetic field, temperature and time.

The Semarkona LL3.0 ordinary chondrite meteorite provides a case study to develop our new methodology. Single grains of the silicate mineral olivine $\left(\mathrm{Mg}_{2} \mathrm{SiO}_{4}\right)$, containing thousands of submicron inclusions of metallic iron, have recently been used to determine the strength of the magnetic field in the early solar nebula [1]. These 'dusty olivine' grains were first measured using a scanning SQUID microscope, enabling their magnetic remanence to be isolated from that of the surrounding meteorite matrix. Using holography and Fresnel-mode Lorentz imaging we confirmed the dominance of single vortex (SV) states in the majority of the remanence carriers (Fig $1 \mathrm{~b}$ and 1c). In-field measurements demonstrated the high stability of this SV state, making them suitable carriers of paleomagnetic information. Focused Ion Beam nanotomography (FIBnT) was then used to reconstruct a $10 \mu \mathrm{m} \times 13 \mu \mathrm{m} \times 5.6 \mu \mathrm{m}$ region from a single grain of dusty olivine (Fig 2a). By acquiring backscattered electron images for each $20 \mathrm{~nm}$ slice, we produced a quantitative reconstruction of the size, shape, orientation and distribution of magnetic Fe nanoparticles in the dusty olivine. This data was then used to produce highly detailed finite element micromagnetic models of individual particles, as well as a micromagnetic reconstruction of the entire ensemble.[2, 3]. This 'nanopaleomagnetic' approach demonstrates for the first time how correlative microscopy, linking information obtained at the 
nano-micro-mm scale, can be used to obtain a physical understanding of the paleomagnetic signals carried by natural materials over 4.5 billion years old.

\section{References:}

[1] R. R. Fu, et al, Science, 346 (2014), p. 108.

[2] T. P. Almeida, et al, Geophys. Res. Lett., 41 (2014), p. 1.

[3] R. J. Harrison and I. Lascu, Geochemistry, Geophys. Geosystems, 15 (2014), p. 1.

[4] The authors acknowledge funding from the ERC under ERC grant agreement 320750-

Nanopaleomagnetism and ERC grant agreement 291522 - 3DIMAGE.
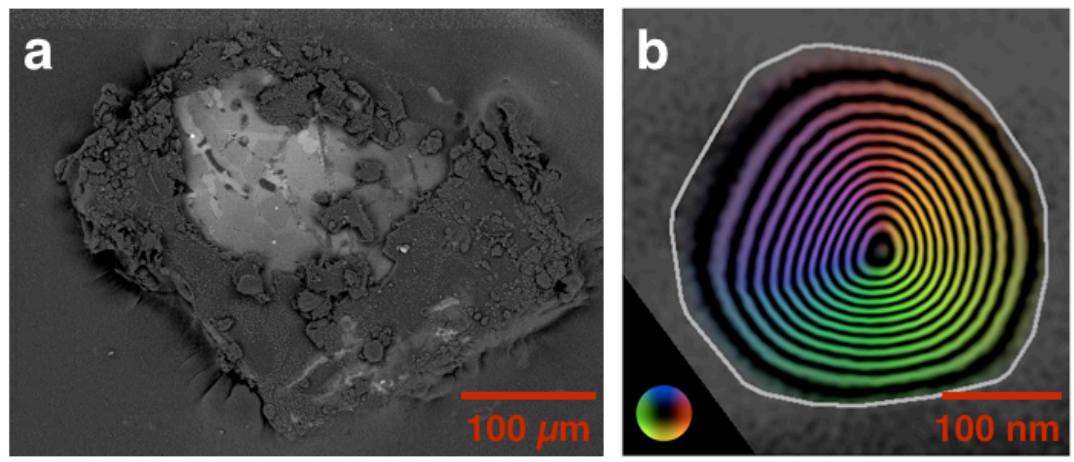

Figure 1. (a) SEM micrograph of the single grain of dusty olivine being studied. Rough dark gray material is mounting epoxy, while bright regions are the exposed olivine. (b) Magnetic induction map for a single $200 \mathrm{~nm}$ Fe particle in the vortex state.
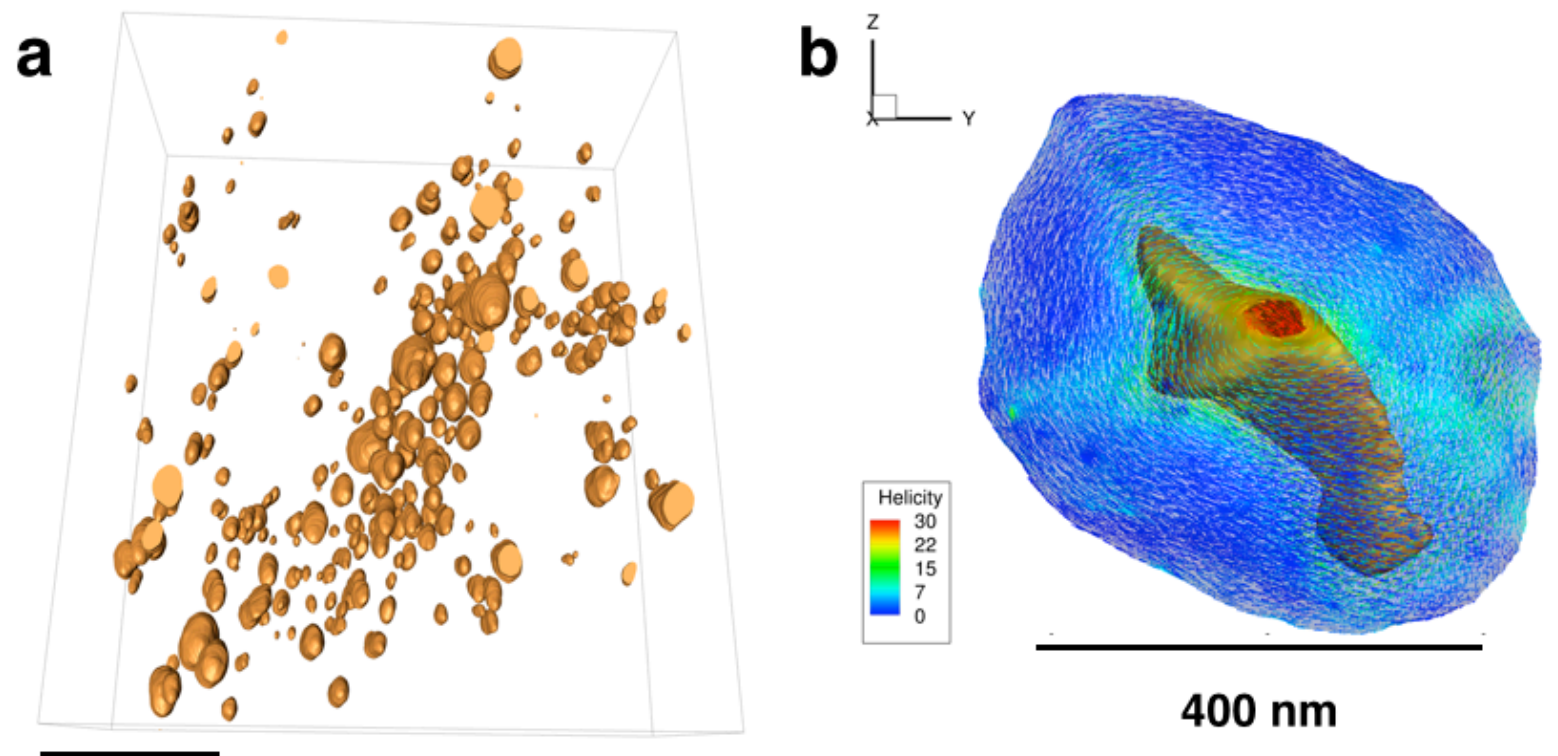

\section{$2.5 \mu \mathrm{m}$}

Figure 2. (a) 3D reconstruction of Fe nanoparticles for dusty olivine grain. (b) FEM modeling results for $400 \mathrm{~nm}$ Fe particle closest to the prolate population mean. Color scale shows helicity of magnetic field. 